\title{
Assimilation of ocean sea-surface height observations of mesoscale eddies ${ }^{\circledR}$
}

Cite as: Chaos 27, 126803 (2017); https://doi.org/10.1063/1.4986088

Submitted: 01 June 2017 . Accepted: 30 October 2017 . Published Online: 14 November 2017

Jeffrey B. Weiss (iD), and lan Grooms (iD)

\section{COLLECTIONS}

EP This paper was selected as an Editor's Pick
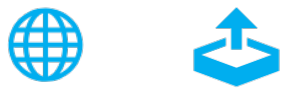

\section{ARTICLES YOU MAY BE INTERESTED IN}

Prediction of flow dynamics using point processes

Chaos: An Interdisciplinary Journal of Nonlinear Science 28, 011101 (2018); https:// doi.org/10.1063/1.5016219

Using periodic orbits to compute chaotic transport rates between resonance zones Chaos: An Interdisciplinary Journal of Nonlinear Science 27, 113104 (2017); https:// doi.org/10.1063/1.4998219

Ocean eddies and climate predictability

Chaos: An Interdisciplinary Journal of Nonlinear Science 27, 126902 (2017); https:// doi.org/10.1063/1.4990034

\section{Don't let your writing keep you from getting published!}

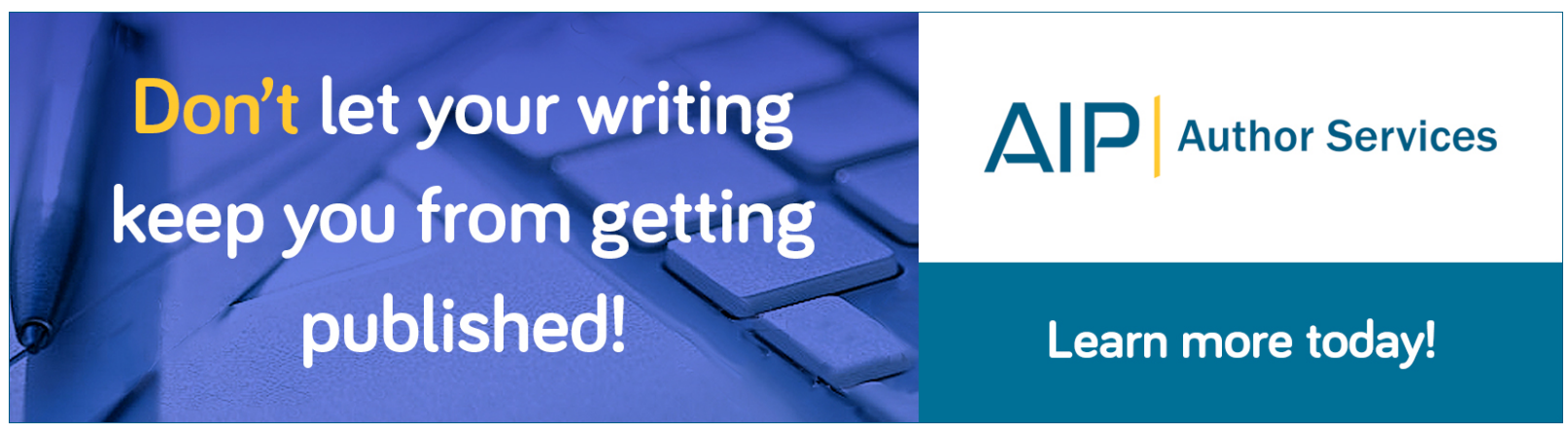




\title{
Assimilation of ocean sea-surface height observations of mesoscale eddies
}

\author{
Jeffrey B. Weiss ${ }^{1, a)}$ and lan Grooms ${ }^{2}$ \\ ${ }^{1}$ Department of Atmospheric and Oceanic Sciences, University of Colorado, Boulder, Colorado 80309, USA \\ ${ }^{2}$ Department of Applied Mathematics, University of Colorado, Boulder, Colorado 80309, USA
}

(Received 1 June 2017; accepted 30 October 2017; published online 14 November 2017)

\begin{abstract}
Mesoscale eddies are one of the dominant sources of variability in the world's oceans. With eddyresolving global ocean models, it becomes important to assimilate observations of mesoscale eddies to correctly represent the state of the mesoscale. Here, we investigate strategies for assimilating a reduced number of sea-surface height observations by focusing on the coherent mesoscale eddies. The study is carried out in an idealized perfect-model framework using two-layer forced quasigeostrophic dynamics, which captures the dominant dynamics of ocean mesoscale eddies. We study errors in state-estimation as well as error growth in forecasts and find that as fewer observations are assimilated, assimilating at vortex locations results in reduced state estimation and forecast errors. Published by AIP Publishing. https://doi.org/10.1063/1.4986088
\end{abstract}

\begin{abstract}
Atmosphere, ocean, and climate models incorporate observations through the procedure of data assimilation. Data assimilation serves to synchronize atmosphere, ocean, and climate models with the chaotic climate system and provides a best-guess estimate of the state of the system. A ubiquitous feature of the world's oceans is that at any one time, they contain thousands of coherent mesoscale eddies, vortices with sizes of tens to hundreds of kilometers and lifetimes of weeks to years. Mesoscale eddies can be identified from satellite altimetry observations of sea-surface height (SSH). As the resolution of ocean models improves, it is increasingly important to assimilate mesoscale eddies, but the amount of data in the full SSH field renders this computationally expensive. Here, we use an idealized model to investigate strategies of assimilating reduced amounts of data and find that assimilating observations at vortex locations improves the estimation of the ocean state and leads to improved forecasts.
\end{abstract}

\section{INTRODUCTION}

The world's oceans are filled with mesoscale eddies, coherent vortices with length scales on the order of $50 \mathrm{~km}$ and lifetimes of weeks to years. ${ }^{1}$ The mesoscale eddies are responsible for roughly half of the mesoscale kinetic energy, ${ }^{1}$ and the total mesoscale kinetic energy is more than an order of magnitude larger than the mean kinetic energy. ${ }^{2,3}$ By trapping water parcels in their interior, mesoscale eddies play an important role in the global transport of heat, momentum, and tracers such as salinity and carbon. Mesoscale eddies can be identified from satellite altimetry measurements of sea surface height (SSH). A census of mesoscale eddies derived from SSH found over 35000 longlived mesoscale eddies (lifetimes $\geq 16$ weeks) over a timespan of 16 years. ${ }^{1}$ Global coupled atmosphere-ocean models currently do not resolve mesoscale eddies, but the next

\footnotetext{
${ }^{a)}$ Electronic mail: jeffrey.weiss@colorado.edu
}

generation of global ocean models will resolve the largest mesoscale eddies. ${ }^{4}$

Data assimilation is a procedure for incorporating information about the detailed state of a system from observations into numerical models that satisfy the system's underlying physical laws. ${ }^{5-7}$ Broadly speaking, data assimilation plays two roles in atmosphere, ocean, and climate modeling. In forecasting, the goal is to produce a best-guess initial state which a model then uses to produce a forecast, and in stateestimation or reanalysis, observations and models are combined to produce an estimate of the time-evolving historical state of the system. Data assimilation can be viewed as a procedure to synchronize the chaotic dynamics of an imperfect model with the chaotic climate system. ${ }^{8-13}$

The turbulent mesoscale dynamics of the ocean is largely in the quasigeostrophic $(\mathrm{QG})$ regime, where rapid rotation and strong stable stratification are the dominant environmental influences. Quasigeostrophic turbulence selforganizes into coherent vortices which carry the majority of the potential vorticity and are responsible for the majority of the kinetic energy. ${ }^{14,15}$ Furthermore, the chaotic nature of such vortex-dominated turbulence is significantly controlled by the chaotic motion of the vortices. ${ }^{16}$

As ocean models resolve mesoscale eddies, it will become increasingly important to use a data assimilation procedure that assimilates the properties of the eddies. Since errors in coherent vortex properties, such as strength and location, drive the error growth in vortex-dominated turbulence, we expect that assimilating mesoscale eddies will lead to improved estimates of the ocean state.

Data assimilation is computationally expensive. The SSH observations that are typically used to identify mesoscale eddies represent a large amount of data to assimilate. We thus investigate strategies where only a fraction of the data is assimilated and look at how choosing observations coincident with vortex locations affects the error. This is broadly similar to the idea that it is necessary to observe within the unstable manifold of the dynamics; ${ }^{17}$ a method based on observing the unstable directions was developed by 
Trevisan and Uboldi. ${ }^{18,19}$ Our approach is based on the idea that since vortices dominate the dynamics, it should be possible to obtain good results at a fraction of the cost by only assimilating observations of the vortices. Our approach is also related to the coherent-structure-based approach of Beechler et al.; ${ }^{20}$ in that approach, the structure of interest was a jet rather than coherent vortices, and the assimilation algorithm included an extra grid-warping step not used here.

We perform a set of numerical experiments assimilating coherent vortices and investigate the effect on the error in the assimilated state. We work in the perfect-model scenario, where two companion simulations are run with identical numerical models. One simulation is designated as "truth" and is allowed to run freely. The second simulation is designated as the "model" and assimilates observations from the "true" simulation. Errors are defined in terms of the difference between "truth" and "model." In the perfect-model framework, these errors are solely due to errors in the state of chaotic systems with identical dynamics. Errors in an ocean model with respect to the actual ocean will be compounded by model errors. Model errors can be reduced by means of effective parameterizations and accounted for within the data assimilation framework. ${ }^{21-25}$

In Sec. II A, the numerical model is described. We use an idealized two-layer quasigeostrophic (QG) model which captures the most important features of the dynamics of mesoscale eddies. In Sec. II B, we describe the vortex identification algorithm used to determine the location of the vortices. Section II C describes the data assimilation strategies we investigate. In Sec. III, we describe the results of the experiments in state-estimation and forecasting frameworks.

\section{METHODS}

\section{A. Model and dynamics}

The investigation is set in the context of a two-layer, doubly periodic QG model on an $f$-plane forced by an imposed mean shear. The nondimensional governing equations are

$$
\begin{gathered}
\partial_{t} q_{1}+\boldsymbol{u}_{1} \cdot \nabla q_{1}=-\nu_{4} \nabla^{4} q_{1} \\
\partial_{t} q_{2}+\boldsymbol{u}_{2} \cdot \nabla q_{2}=-c_{d} \operatorname{curl}\left[\left|\left(\boldsymbol{u}_{2}+\hat{\boldsymbol{x}}\right)\right|\left(\boldsymbol{u}_{2}+\hat{\boldsymbol{x}}\right)\right]-\nu_{4} \nabla^{4} q_{2} \\
q_{1}=y+\nabla^{2} \psi_{1}+\frac{1}{2}\left(\psi_{2}-\psi_{1}\right) \\
q_{2}=-y+\nabla^{2} \psi_{1}+\frac{1}{2}\left(\psi_{1}-\psi_{2}\right)
\end{gathered}
$$

where $q_{i}$ and $\psi_{i}$ are the potential vorticity and streamfunction, respectively, in layer $i$. The components of $q$ and $\psi$ which are linear in $y$ are associated with an imposed uniform zonal baroclinic shear. The velocity is orthogonal to the gradient of the streamfunction: $\boldsymbol{u}_{i}=\left(-\partial_{y} \psi_{i}, \partial_{x} \psi_{i}\right)$. The term multiplied by $c_{d}$ in Eq. (2) is a standard quadratic drag where the imposed zonal velocity in the lower layer $-\hat{\boldsymbol{x}}$ has been subtracted from $u_{2}$ (which includes the mean flow) before computing the drag. Hyperviscous dissipation is included with identical hyperviscosity coefficients $\nu_{4}$ in each layer. The equations have been nondimensionalized using the imposed zonal velocity as a velocity scale and the deformation radius as a length scale. We set $c_{d}=0.1$ and $\nu_{4}=0.08192$. The domain is square and has nondimensional width $32 \pi$. Approximate solutions are computed using $256 \times 256$ nonzero Fourier modes and a fourth-order semiimplicit Runge-Kutta method as described by Grooms and Majda. ${ }^{26}$ The configuration is exactly as used recently by Grooms and Zanna. ${ }^{27}$ The nondimensional grid size is 0.39 , so there are just more than two grid points per deformation radius, which is sufficient for eddy-resolving computations of this kind. ${ }^{28}$ The nondimensional time step is 0.01 . Figure 1 shows a snapshot of the upper-layer relative vorticity $\nabla^{2} \psi_{1}$ from the eddy-resolving simulation. One sees that the flow is dominated by a population of self-organized coherent vortices, which are the analogue in the QG system of the mesoscale eddies in the ocean.

\section{B. Eddy census and dimensionalization}

A central component of the investigation involves identifying vortex locations in observations and in simulations. We use an algorithm that we developed as a simplified approximation to more complete methods, like the ones used in Refs. 1 and 29. The algorithm is applied to a generic field, either top-layer streamfunction $\psi_{1}$ in the case of observational data or top-layer potential vorticity $q_{1}$ in the case of simulation data.

\section{Vortex Identification Algorithm}

1. Set a threshold equal to twice the RMS (root-meansquare) value of the field.

2. Find all connected regions where the field magnitude is above threshold.

3. For each region

(a) Find the extremum (max or min) of the field within the region. If it lies on the boundary of the region, discard the region.

(b) Find the area of the region. If it is larger than 300 grid points, discard the region.

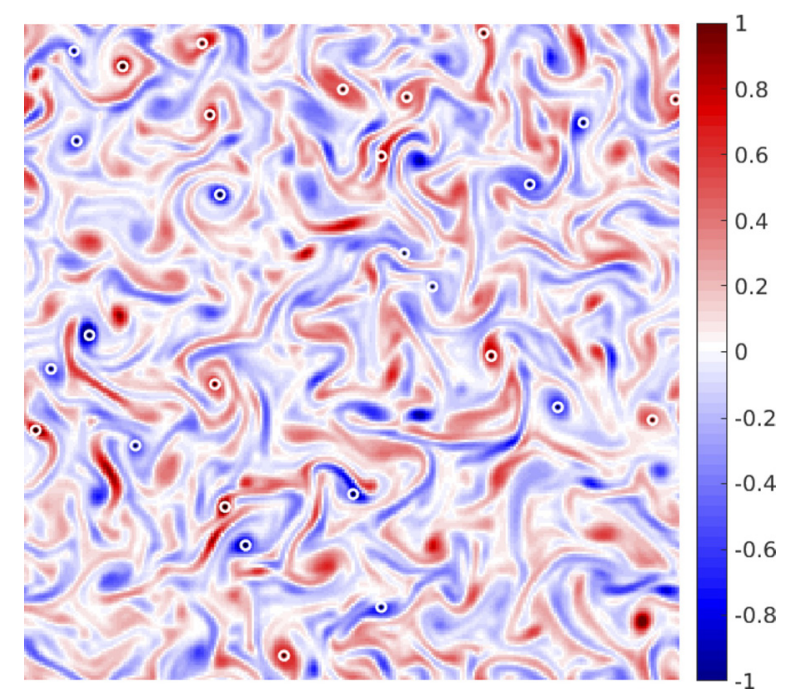

FIG. 1. Snapshot of the upper layer vorticity, in units of $10^{-5} / \mathrm{s}$. White circles represent vortex locations identified by the algorithm described in Sec. II B. 
4. The vortex locations are the locations of the extrema in regions that were not discarded.

The algorithms in Refs. 1 and 29 are more complex; e.g., they re-apply the above algorithm using a range of different thresholds in step 1, and they include conditions on the shape of the region above the threshold.

The locations of eddies identified by this algorithm are shown in Fig. 1. There are clearly vortices which have been missed by the algorithm. This is in part due to the simplified nature of the algorithm but is more closely tied to the fact that the locations are identified using $\psi_{1}$ rather than the vorticity shown in Fig. 1; more vortices are identified when the algorithm is applied to the latter. The more complex algorithms are in practice applied to SSH (analogous to $\psi_{1}$ ) rather than vorticity because the differentiation needed to compute vorticity amplifies any observation errors. There is no such difficulty in the vorticity field obtained from the model, so the vorticity field is used to obtain the locations of vortices in the model data.

To examine the statistics of eddies in the simulation, the above algorithm was applied to $\psi_{1}$. Each identified vortex was extracted, and the vortices were averaged. The averaged vortex was approximately circular, with a maximum nondimensional velocity of 18 at a radius of 5 grid points. Reasonable agreement with the vortex statistics identified by Chelton et al. ${ }^{1}$ in global observations of sea surface height was obtained by assigning a dimensional value of $15 \mathrm{~km}$ to the spatial grid and a dimensional value of $1 \mathrm{~cm} / \mathrm{s}$ to the velocity. The average dimensional vortex radius is thus $75 \mathrm{~km}$, the dimensional deformation radius is $38.2 \mathrm{~km}$, the dimensional domain width is $3,840 \mathrm{~km}$, and the dimensional time scale is approximately 42 days ( 6 weeks). This approach to assigning dimensional values to two-layer quasigeostrophic simulations, based on vortex statistics, is in contrast to more ad hoc approaches based on bulk enstrophy. ${ }^{23,30}$ The data assimilation described below is carried out using a three-year simulation with data saved weekly.

\section{Data assimilation}

In this section, we describe details of the assimilation system. The observation models mimicking sea surface height (SSH) and sub-surface density observations are presented first. Next, we present the details of the assimilation algorithm itself, which is based on a static background covariance. Finally, we present the details of the coherentvortex-based assimilation algorithm, which chooses to assimilate a subset of the SSH observations based on vortex locations.

We consider the assimilation of satellite observations of sea surface height (SSH) into eddy-resolving models. These observations are plentiful, and the observations from a flock of satellites are regularly processed to a weekly, gridded $\mathrm{SSH}$ data product. ${ }^{31} \mathrm{Xu}$ and $\mathrm{Fu}^{32}$ and Dufau et al. ${ }^{33}$ examine the error statistics of along-track satellite SSH observations. They find that the errors are approximately white, with an amplitude level such that the error spectrum intersects the SSH spectrum at a wavelength of $40-65 \mathrm{~km}$, depending on the satellite. We find that the spectrum of Gaussian white noise with a variance of $0.25^{2}$ (nondimensional) intersects with the spectrum of $\psi_{1}$ at a dimensional wavelength of $65 \mathrm{~km}$.

To crudely approximate the smoothing effect that the processing from along-track to weekly gridded data product has on the error, we first add white noise with a standard deviation of 0.25 to $\psi_{1}$ and then apply a moving-average convolution. The convolution is implemented by tapering the Fourier coefficients: coefficients of modes with wavelengths smaller than $30 \mathrm{~km}$ are set to zero, modeling the $1 / 4^{\circ}$ grid resolution of the gridded data product. Coefficients of modes with wavelengths larger than $364 \mathrm{~km}$ are left unchanged, modeling the effective resolution of the data product estimated by Chelton et al. ${ }^{1}$ Coefficients of modes with wavelengths between 30 and $364 \mathrm{~km}$ are multiplied by a linear function of wavenumber. SSH observations are assimilated weekly, i.e., every $1 / 6$ nondimensional time units.

In summary, synthetic SSH observations are generated by first adding white noise to $\psi_{1}$ and then smoothing the result so that wavelengths larger than $364 \mathrm{~km}$ are unchanged while wavelengths down to $30 \mathrm{~km}$ are reduced. The observation errors are the difference between the true $\psi_{1}$ and the synthetic observations. Because of the smoothing, the observations have no information at the smallest scales.

The above approach generates synthetic gridded SSH observations whose errors are analogous to the errors in the gridded ocean SSH observations. The errors are spatially correlated with a variance of 0.083 greater than the nominal value of $0.25^{2}$. Our data assimilation algorithm takes the spatial correlation of the observation errors into account, so we estimated the spatial covariance structure using the threeyear long weekly dataset.

The ARGO array is the primary source of sub-surface observational data. ${ }^{34}$ The ARGO array consists of floats that, approximately every 10 days, observe density along a vertical profile in the top two kilometers of the ocean. The typical distance between floats is on the order of $300 \mathrm{~km}$. We model ARGO profiles by observing the difference in streamfunction between the two layers, $\psi_{1}-\psi_{2}$, on an equispaced grid with a spacing of $240 \mathrm{~km}$. Unlike the real ARGO floats, the grid is fixed-it does not move according to the model dynamics. ARGO observations have very small errors (nondimensional variance: $2.5 \times 10^{-5}$ ), and the errors are uncorrelated in space. As with the SSH data, our synthetic ARGO data are assimilated weekly. While these idealized floats do not capture all the features of the actual ARGO observing system, they allow us to include a realistic amount of sub-surface data and thus provide a balance to the surface SSH observations.

A single set of observations is generated for every week of the three year assimilation cycle. All of the assimilation experiments presented here use the same set of observations. We use optimal interpolation as the data assimilation method. $^{7}$ Optimal interpolation is used because it is less computationally costly than ensemble methods. Eddyresolving ocean models are computationally expensive enough that it is difficult to run even modestly sized ensembles for data assimilation; optimal interpolation is less 
expensive and balances the high cost of model simulations. The methods described below could equally well be implemented in a 3D-Var framework.

Let $\boldsymbol{x}_{k}$ denote the model state variable $\psi$ on the computational grid at assimilation cycle $k$, and let $\boldsymbol{y}_{k}$ denote the vector of observations used in the assimilation at assimilation cycle $k$. The state and observations are related by an observation matrix $\mathbf{H}_{k}$, and the observation errors are described above. The data assimilation algorithm proceeds as follows. First, a one-week forecast is made, denoted by $\boldsymbol{x}_{k}^{-}$. Then, the observations $\boldsymbol{y}_{k}$ are assimilated as follows:

$$
\boldsymbol{x}_{k}=\boldsymbol{x}_{k}^{-}+\mathbf{K}_{k}\left(\boldsymbol{y}_{k}-\mathbf{H}_{k} \boldsymbol{x}_{k}^{-}\right) .
$$

This creates $\boldsymbol{x}_{k}$, which is the "analysis," and is used as the initial condition for the next one week forecast. The first initial condition is provided by the true state at the end of the three year assimilation window, making the initial condition completely decorrelated from the true state at the beginning of the window. The matrix $\mathbf{K}_{k}$ is the Kalman gain matrix

$$
\mathbf{K}_{k}=\mathbf{C} \mathbf{H}_{k}^{T}\left(\mathbf{H}_{k} \mathbf{C} \mathbf{H}_{k}^{T}+\mathbf{R}_{k}\right)^{-1},
$$

where $\mathbf{R}_{k}$ is the covariance matrix of the observation errors associated with $\boldsymbol{y}_{k}$.

The time-independent "background" covariance C describes the forecast uncertainty. The background covariance is modeled as a homogeneous, isotropic random field with "square exponential" correlation function: ${ }^{35}$ the correlation between points separated by a distance $\delta$ is $e^{-\|\delta\|^{2} / L_{c}^{2}}$ where the correlation length $L_{c}=105 \mathrm{~km} .{ }^{36}$ The upper-layer variance is $1.6^{2}$ (nondimensional), and the lower layer variance is $1.1^{2}$. The cross-correlation between the upper and lower layers is also "square exponential" with a correlation coefficient of 0.78 . This background covariance is an infinitely smooth limit of the multivariate Matern model, ${ }^{37}$ which is appropriate to fields like $\psi$ that have a rapidly decaying spectrum.

We compare three assimilation strategies: grid-based, vortex-based, and hybrid. All strategies assimilate all of the ARGO data; the strategies are distinguished by the way SSH observations are used. The grid-based assimilation uses an equispaced grid of observations. We approximate the observation error covariance matrix $\mathbf{R}_{k}$ as diagonal; this is a good approximation for coarse observing grids and is driven by convenience for fine grids.

The vortex-based strategy assimilates observations only near vortices. At each assimilation cycle, the vortexidentification algorithm is applied to both the observations and the model forecast. The vortex locations are identified directly from the SSH observations, while for the model forecast, the identification is based on potential vorticity rather than $\psi_{1}$. This results in two lists of vortex locations, which contain some overlap. To eliminate duplicates, any forecast location that is less than 5 grid points $(75 \mathrm{~km})$ from an observation location is eliminated. Having thus created a single list of vortex locations, a subset of SSH observations is selected to be used in the assimilation.

We compared four different vortex observation configurations. The first uses only the SSH observation at the center of each vortex. The next uses 5 SSH observations per vortex: the center point plus points 4 grid points $(75 \mathrm{~km})$ north, south, east, and west of the center. The next uses 9 SSH observations per vortex: all the 5-point locations plus the four corner points. The fourth uses 16 SSH observations per vortex: a $4 \times 4$ grid with a spacing of $60 \mathrm{~km}$, centered on the vortex location. These four configurations are shown in Fig. 2.

Since the observation locations over a single vortex are close enough that their errors are not uncorrelated, we use an estimate of the observation error covariance within the assimilation over a single vortex; observation errors for different vortices are assumed uncorrelated. As a result, $\mathbf{R}_{k}$ is block-diagonal, with diagonal blocks corresponding to different vortices. The estimate of observation error correlation over a single vortex is obtained by a Monte-Carlo estimate: synthetic observations are generated over vortices and subtracted from the true field to obtain samples of the observation error. Many such samples are generated and are used to estimate the observation error covariance matrix associated with a single vortex.

The final strategy is a hybrid between the grid-based and vortex-based strategies. There can be large gaps where no vortices exist, and it is helpful to assimilate observations at locations within these regions. The hybrid strategy thus augments the list of vortex locations with the locations of points on a $16 \times 16$ grid of points with a spacing of $240 \mathrm{~km}$. Duplicate locations are eliminated so that points on the grid are not assimilated if they are near vortex locations.

\section{RESULTS}

Subsections III A and III B present results for state estimation and forecasting for the three assimilation strategies: grid, vortex, and hybrid. A key aspect of the comparison is the number of SSH observations used in each strategy (since all methods use the same ARGO data). The number of SSH

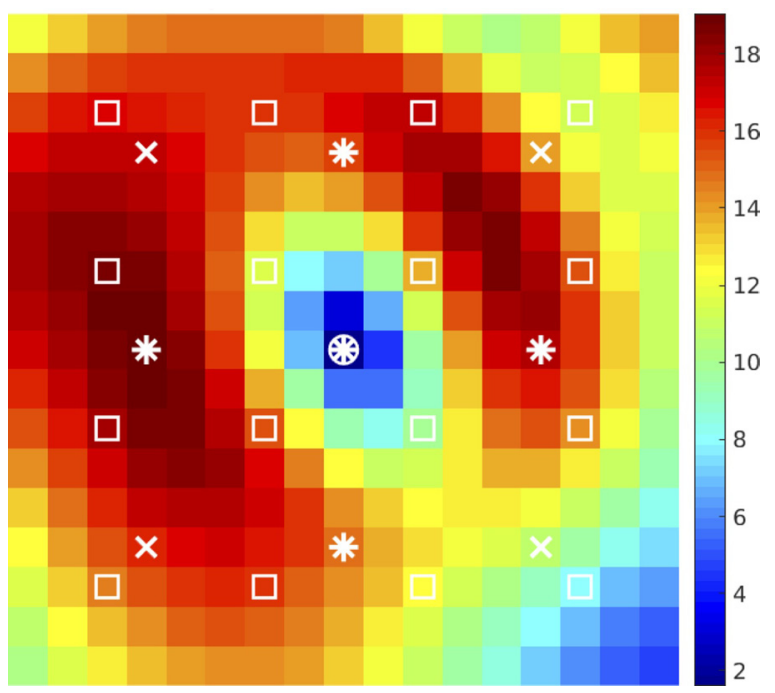

FIG. 2. Four vortex observation grids. The colorscale shows the amplitude of surface velocity $\left|\boldsymbol{u}_{1}\right|$ for a typical eddy (nondimensional, equiv. cm/s). The circle at the center shows the identified center of the vortex. Locations marked with + are the 5-point assimilation grid. Locations marked with$\times$ are the 9-point assimilation grid, and locations marked with a square are the 16-point grid. 
RMS Streamfunction Errors

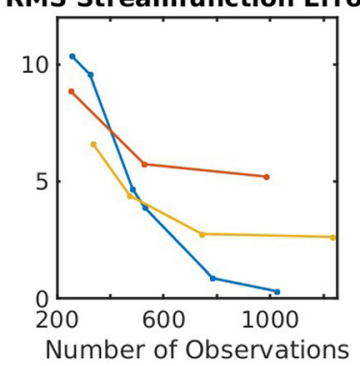

RMS Velocity Errors

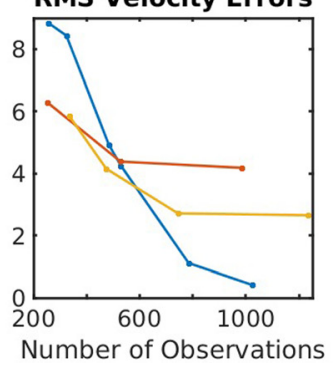

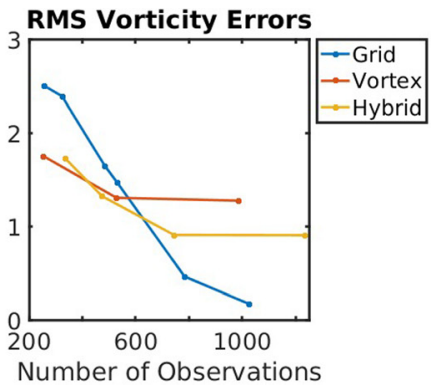

FIG. 3. The RMS (root mean square) assimilation error for streamfunction (left, Sv), velocity (center, $\mathrm{cm} / \mathrm{s}$ ), and relative vorticity (right, $10^{-6} / \mathrm{s}$ ) as a function of the average number of SSH observations assimilated per week. The units of streamfunction are Sverdrups; $1 \mathrm{~Sv}=10^{6} \mathrm{~m}^{3} / \mathrm{s}$. observations assimilated per one-week assimilation cycle is constant in the grid-based approach, while in the vortexbased and hybrid approaches, the number of observations assimilated per cycle depends on the number of vortices identified per cycle. Thus, we compare the methods on the basis of the average number of SSH observations assimilated per cycle over the three-year window, which is main driver of the computational cost. Note that the domain is a $3840 \mathrm{~km}$ square, so the number of observations can be converted to an observation density; e.g., 512 observations become approximately one observation per $170^{2} \mathrm{~km}^{2}$. Before presenting the results, we briefly note that assimilating only ARGO data (without $\mathrm{SSH}$ ) leads to poor results, which is not surprising given the coarse spatial resolution of the ARGO data.

The state errors are defined in terms of three different error norms: the RMS streamfunction, velocity, and relative vorticity errors. These three quantities all have the same information content, but because velocity is a derivative of streamfunction and relative vorticity is a derivative of velocity, the three norms provide measures of the error that emphasize different length scales: streamfunction is sensitive to the larger scales, velocity is sensitive to intermediate scales, and vorticity is sensitive to the smaller scales. One measure that quantifies this scale-sensitivity is the integral scale of the field. (The "integral" scale is defined in terms of the power spectrum of a field $P(k)$, where $k$ is the magnitude of the wavenumber; the integral scale is $\int P(k) \mathrm{d} k / \int k P(k)$ $\mathrm{d} k$.) The integral scale for streamfunction is $114 \mathrm{~km}$, for velocity, it is $60 \mathrm{~km}$, and for vorticity, it is $31 \mathrm{~km}$.

\section{A. State estimation}

In this section, we present the state estimation performance, i.e., we present measures of the error between the true system state and the data-assimilated estimated state averaged over the time evolving state trajectory. The initial condition for the estimated state is taken to be the "true" state at the end of the three year assimilation window. This provides an initial state for the assimilation that has the same statistics as the "true" state but is completely decorrelated from the true state at the beginning of the window.

Figure 3 shows the errors in streamfunction, velocity, and vorticity as a function of the average number of observations assimilated per week. In Fig. 3, the errors are squared and averaged across the domain, across the two layers, and across the last year of the simulation; the square root of this average is displayed as the RMS (root mean square) error.

The average number of observations assimilated per week in the vortex and hybrid methods depends on the number of observations assimilated per vortex. The results for the vortex-based method with a single observation per vortex are quite poor and are not shown. For the vortexbased method, the first data point on the line in Fig. 3 is for the 5-point vortex observation grid, the second point on the line is for the 9-point grid, and the third point is for the 16point grid. For the hybrid method, the points refer to the 1point, 5-point, 9-point, and 16-point vortex observation grids.

As the number of grid points increases, the errors decrease, and for the largest number of observations per week $(\sim 1000)$, the grid-based method is clearly the best. On the other hand, for less than about 500 observations per cycle, the vortex-based and hybrid methods are superior. In particular, when using a small number of observations, the vortex-based method does the best job of estimating the vorticity field, i.e., the smallest scales of the flow, the vortex-based and hybrid methods have similar errors for the velocity, i.e., intermediate scales, while the hybrid method is best for the streamfunction, i.e., the larger scales. As the number of observations increases (by increasing the number of observations per vortex), the performance of the vortex-based method reaches a plateau. This is presumably because the 9point vortex observation grid is sufficient to resolve the vortex and the extra observations in the 16-point vortex grid are redundant; the cost of these observations would be more effectively spent observing regions without vortices. This behavior was the motivation for the development of the hybrid approach.

Given the clear superiority of the vortex/hybrid vs. gridbased methods for small vs. large numbers of observations, we focus in what follows on the region where the results are similar. We thus present results for the grid-based method with $21^{2}=484$ points and a spacing of $165 \mathrm{~km}$, for the vortex-based method with 9 points per vortex which has an average of 528 observations per week, and for the hybrid method with a grid spacing of $240 \mathrm{~km}$ and 5 points per vortex for an average total of 474 observations per week.

We next investigate the rate at which the error is reduced by the three assimilation strategies after starting from a model state which is uncorrelated with the "truth." Figure 4 shows the RMS error as a function of time (here, the errors are squared and averaged over space and over the layers), for the three physical fields and for the three methods. Some point of reference is useful for understanding the magnitude of the errors. If there were no data assimilation, the squared error would be twice the climatological variance; similarly, if the state estimate were simply equal to the climatological mean (which is zero here), then the squared error would be equal to the climatological variance. In each 

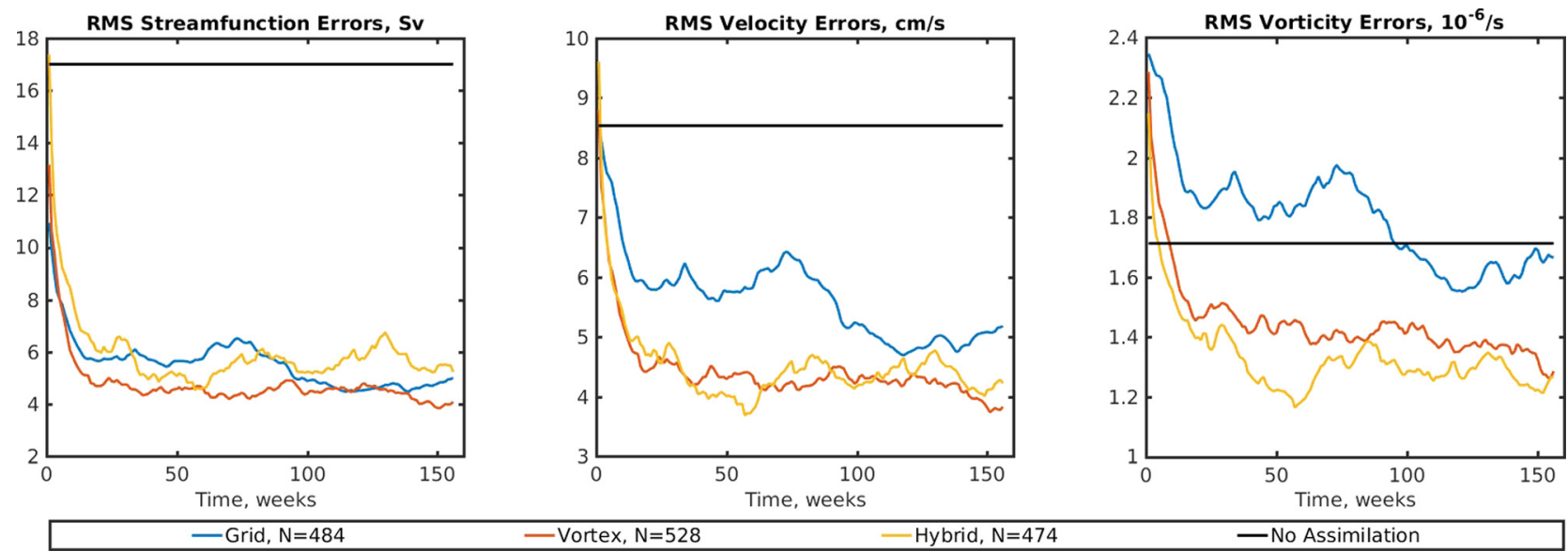

FIG. 4. The RMS error in streamfunction (left, Sv), velocity (center, $\mathrm{cm} / \mathrm{s}$ ), and relative vorticity (right, $10^{-6} / \mathrm{s}$ ) as a function of time for the three methods with three specific configurations: grid-based with 484 observations per week, vortex-based with an average of 528 observations per week ( 9 per vortex), and hybrid with an average of 474 observations per week (5 per vortex plus a $240 \mathrm{~km}$ grid). The black line shows the square root of the climatological variance.

panel of Fig. 4, the black line shows the square root of the climatological variance.

The streamfunction error decreases very rapidly with time for all three methods and equilibrates at similar levels after three to six months. The velocity and vorticity errors also decrease rapidly and then perhaps show signs of further slow improvement. It is worth noting that the initial rate of decrease of velocity and vorticity error is faster in the vortex and hybrid methods than in the grid-based method; although the final error levels are similar, the vortex and hybrid methods synchronize more quickly. Also, at this density of observations, it takes the grid-based method almost two years to achieve the same level of vorticity errors as would be achieved by simply replacing the state estimate with the climatological mean (which is zero).

\section{B. Forecasting}

While the RMS errors in state estimation are important, they do not give a complete picture of the accuracy of forecasts made using the state estimate as an initial condition. To measure forecast accuracy, we ran 8-week forecasts starting from each of the weekly state estimates. Figure 5 shows as a solid black line the RMS velocity errors in the hybrid state estimate as a function of time; the dashed black lines show the RMS errors in 8-week forecasts initialized every 13 weeks from the state estimate. These forecast errors grow approximately exponentially as expected for a chaotic system.

We averaged the forecast errors starting from weekly state estimates over the last year of the assimilation window for the three methods. Figure 6 shows the average growth of the forecast error for the three physical fields and for the three strategies.

The behavior of the forecast error for streamfunction is an excellent example of the differences between the strategies. Although the initial (state-estimate) error is lower for the grid-based method than for the vortex-based method, the forecasts from the vortex-method become more accurate than those from the grid-based method for lead times longer than 4 weeks. The fact that the error grows more slowly in the vortex-based method shows that the initial, state-estimate error in the unstable subspace is smaller for the vortex-based method than for the grid-based method.

In velocity forecasting, all three methods have similar error growth rates, and differences in forecast error seem to be primarily due to differences in the initial, state-estimate error, which is governed by the method and the number of observations.

The grid-based method does a poor job of forecasting vorticity, with an initial error level near the climatological error. The error then grows slowly towards the error level that would be expected for a simulation with no data assimilation, i.e., the climatological error multiplied by $\sqrt{2}$. The vortex-based method does the best job of forecasting vorticity, slightly better than the hybrid method. The growth of vorticity errors in the forecast for the vortex-based and hybrid methods is similar, and the forecasts reach climatological error levels after about 5 weeks.

\section{DISCUSSION AND CONCLUSION}

Motivated by the problem of data assimilation in ocean models which resolve mesoscale eddies, we investigated

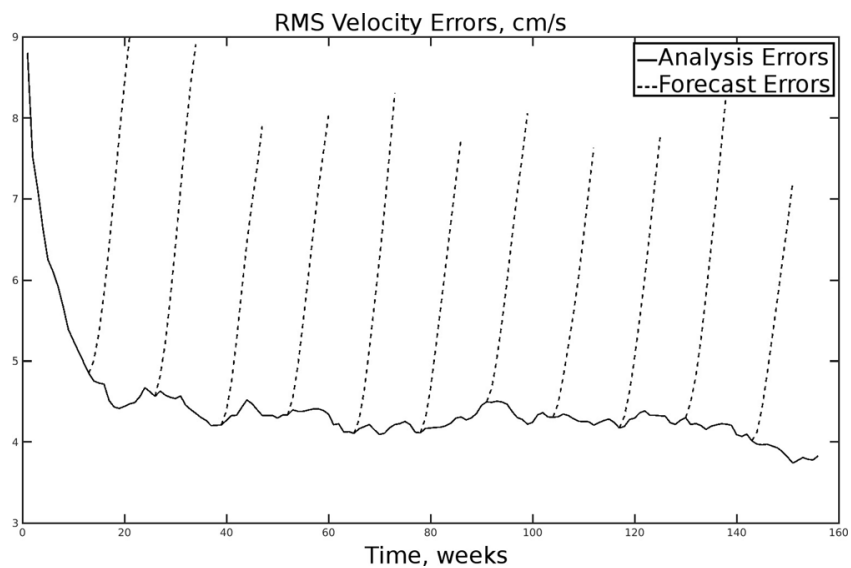

FIG. 5. The solid black line shows the RMS velocity errors $(\mathrm{cm} / \mathrm{s})$ in the state estimate of the hybrid method as a function of time. The dashed lines show the growth of the RMS errors in 8-week forecasts initialized from the state estimate. 

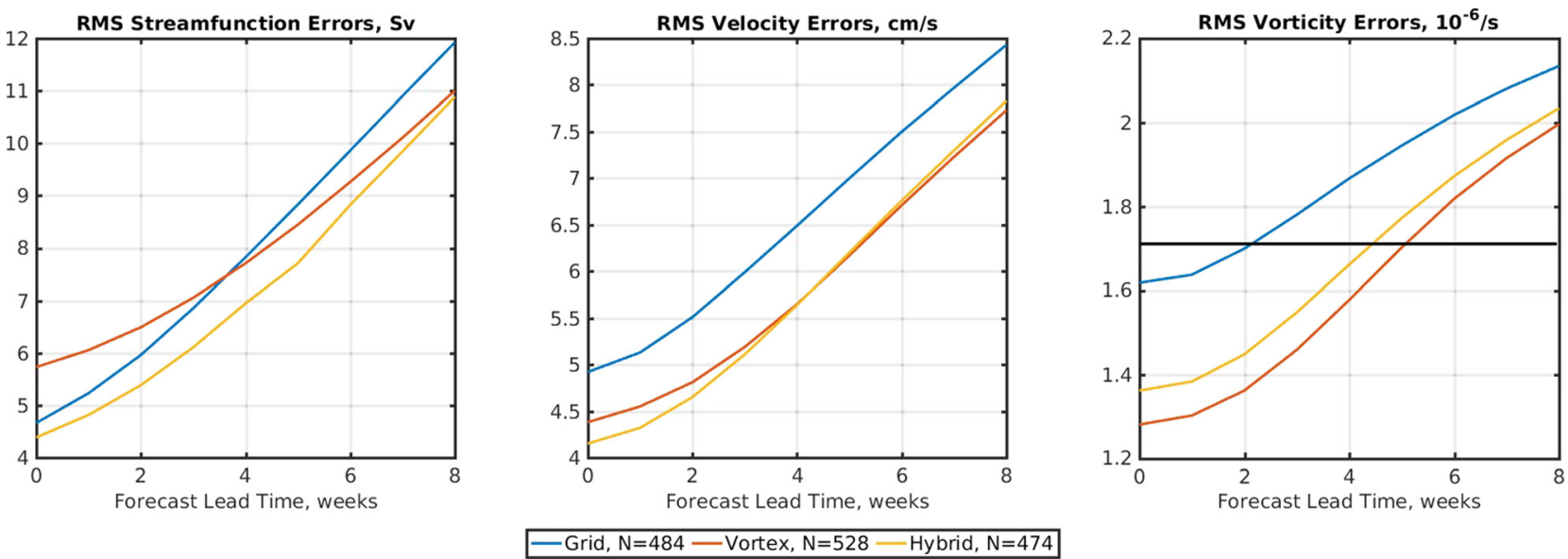

FIG. 6. RMS Forecast errors in streamfunction (left, Sv), velocity (center, $\mathrm{cm} / \mathrm{s}$ ), and relative vorticity (right, $10^{-6} / \mathrm{s}$ ) as a function of forecast lead time. The black line in the right panel shows the square root of the climatological vorticity variance. The three methods are the grid-based method with $484=22^{2}$ observations per week, the vortex-based method with an average of 528 observations per week ( 9 per vortex), and the hybrid method with an average of 474 observations per week ( 5 per vortex plus a $240 \mathrm{~km}$ grid).

strategies to assimilate the flow in two-layer forced quasigeostrophic vortex turbulence. The observational strategy is designed to be analogous to ARGO floats and satellite SSH observations. Naturally, excellent results are achieved when a very dense set of observations are assimilated. This, however, is computationally expensive, and we focus on whether good results can be achieved by assimilating only a judiciously chosen subset of observations. We find that an approach that simply assimilates a subset of observations on a regular grid is less effective than methods that assimilate observations taken over coherent mesoscale vortices.

Chaotic synchronization is effected by providing information about the state of the system along the unstable manifold of the chaotic dynamics. Studies of vortex dominated turbulence suggest that the chaotic motion of the vortices drives the chaotic error growth (e.g., Ref. 16). The success of assimilating vortex locations indicates that the vortex locations provide important information about the unstable manifold in vortex dominated turbulence.

The vortex-based methods improve as the number of observations per vortex increases; however, the improvement slows once a vortex is sufficiently observed. Assimilating 9 observations in a $150 \mathrm{~km}$ square centered on each vortex has a better ratio of accuracy to cost than assimilating 16 points per vortex or 5 points per vortex. Also, since there are large regions without vortices, a hybrid approach that assimilates both over vortices and on a sparse $240 \mathrm{~km}$ grid leads to improvements compared to a purely vortex-based approach.

We investigated how the error in state estimation depends on the number of observations. If the observations are dense enough, then assimilating on a grid is the best method. For sparse observations, the hybrid method generally performs better, although for very few observations, the vortex method does a better job in estimating the vorticity field. Thus, if it is computationally too expensive to assimilate SSH on a fine grid, then it is advantageous to use the hybrid method.

The vortex-based approaches are more efficient than assimilating on a $240 \mathrm{~km}$ grid and less efficient than assimilating on a $120 \mathrm{~km}$ grid (which has 4 times more observations and therefore costs significantly more). At an intermediate grid density, the state-estimation errors of the grid-based and vortex-based errors are comparable.

We then compared how long it takes the error to converge following weekly assimilation using the number of observations that result in similar converged errors. All scales converge similarly for the vortex and hybrid methods. The larger scales, as indicated by the streamfunction error, converge similarly for the three methods. The intermediate and smaller scales show significantly more rapid convergence for the vortex and hybrid methods compared to the grid method.

Finally, we investigated the error growth in forecasts begun with initial states created by the three assimilation methods using the number of observations where the errors are roughly the same size. Forecasts initialized from the state estimates from the vortex-based methods had slower error growth in the rms streamfunction error than forecasts initialized from the grid-based method. The rms velocity errors had similar growth rates while the rms vorticity errors grew slower for the grid-based method, but for the grid-based method, these errors were significantly larger at all times. This indirectly demonstrates that the vortex-based methods result in state estimates with less error in the unstable subspace than the grid-based method.

By restricting ourselves to QG flow in a perfect-model scenario, we have focused on the scenario where state errors are dominated by the chaotic motion of the vortices. The ocean has other flow features that are not captured by the QG approximation and the particular domain and forcing we use, such as mean gyres, western boundary currents, and submesoscale dynamics. These will lead to additional errors in assimilating ocean observations. Model error in ocean models leads to another source of error in ocean data assimilation. Yet given the important role that mesoscale eddies play in the ocean kinetic energy, we expect that our results will carry over and that assimilating mesoscale eddies will lead to improvements in assimilating observations into eddyresolved ocean models. 


\section{ACKNOWLEDGMENTS}

J.B.W. was partially supported by NSF-OCE-1245944. J.B.W. would like to thank Gregory S. Duane for helpful conversations about coherent structures and chaotic synchronization. I.G. would like to thank Alicia Karspeck for helpful conversations about data assimilation in eddyresolving global ocean models.

${ }^{1}$ D. B. Chelton, M. G. Schlax, and R. M. Samelson, "Global observations of nonlinear mesoscale eddies," Prog. Oceanogr. 91, 167-216 (2011).

${ }^{2}$ K. Wyrtki, L. Magaard, and J. Hager, "Eddy energy in the oceans," J. Geophys. Res. 81, 2641-2646, https://doi.org/10.1029/JC081i015p02641 (1976).

${ }^{3}$ P. L. Richardson, "Eddy kinetic energy in the north atlantic from surface drifters," J. Geophys. Res. 88, 4355-4367, https://doi.org/10.1029/ JC088iC07p04355 (1983).

${ }^{4}$ B. Pearson, B. Fox-Kemper, S. Bachman, and F. Bryan, "Evaluation of scale-aware subgrid mesoscale eddy models in a global eddy-rich model," Ocean Model. 115, 42-58 (2017).

${ }^{5}$ R. Daley, Atmospheric Data Analysis (Cambridge University Press, 1991).

${ }^{6} \mathrm{M}$. Ghil and P. Malanotte-Rizzoli, "Data assimilation in meteorology and oceanography," Adv. Geophys. 33, 141-266 (1991).

${ }^{7}$ E. Kalnay, Atmospheric Modeling, Data Assimilation and Predictability (Cambridge University Press, 2003).

${ }^{8}$ G. S. Duane and J. J. Tribbia, "Synchronized chaos in geophysical fluid dynamics," Phys. Rev. Lett. 86, 4298-4301 (2001).

${ }^{9}$ G. S. Duane, J. J. Tribbia, and J. B. Weiss, "Synchronicity in predictive modelling: A new view of data assimilation," Nonlinear Proc. Geophys. 13, 601-612 (2006).

${ }^{10}$ S.-C. Yang, D. Baker, H. Li, K. Cordes, M. Huff, G. Nagpal, E. Okereke, J. Villafae, E. Kalnay, and G. S. Duane, "Data assimilation as synchronization of truth and model: Experiments with the three-variable lorenz system," J. Atmos. Sci. 63, 2340-2354 (2006).

${ }^{11} \mathrm{Z}$. Toth and M. Peña, "Data assimilation and numerical forecasting with imperfect models: The mapping paradigm," Physica D 230, 146-158 (2007).

${ }^{12}$ G. S. Duane and J. J. Tribbia, "Dynamical synchronization of truth and model as an approach to data assimilation, parameter estimation, and model learning," in Nonlinear Dynamics in Geosciences (Springer New York, New York, 2007) pp. 291-310.

${ }^{13}$ A. Carrassi, M. Ghil, A. Trevisan, and F. Uboldi, "Data assimilation as a nonlinear dynamical systems problem: Stability and convergence of the prediction-assimilation system," Chaos 18, 023112 (2008).

${ }^{14}$ J. C. McWilliams, J. B. Weiss, and I. Yavneh, "Anisotrophy and coherent vortex structures in planetary turbulence," Science 264, 410-414 (1994).

${ }^{15}$ J. C. McWilliams, J. B. Weiss, and I. Yavneh, "The vortices of homogeneous geostrophic turbulence," J. Fluid Mech. 401, 1-26 (1999).

${ }^{16}$ A. Babiano, G. Boffetta, A. Provenzale, and A. Vulpiani, "Chaotic advection in point vortex models and twodimensional turbulence," Phys. Fluids 6, 2465-2474 (1994).

${ }^{17}$ K. Law, A. Stuart, and K. Zygalakis, Data Assimilation: A Mathematical Introduction (Springer, 2015).

${ }^{18}$ A. Trevisan and F. Uboldi, "Assimilation of standard and targeted observations within the unstable subspace of the observation-analysis-forecast cycle system," J. Atmos. Sci. 61, 103-113 (2004).
${ }^{19} \mathrm{~F}$. Uboldi and A. Trevisan, "Detecting unstable structures and controlling error growth by assimilation of standard and adaptive observations in a primitive equation ocean model," Nonlinear Proc. Geophys. 13, 67-81 (2006).

${ }^{20}$ B. E. Beechler, J. B. Weiss, G. S. Duane, and J. Tribbia, "Jet alignment in a two-layer quasigeostrophic channel using one-dimensional grid warping," J. Atmos. Sci. 67, 2296-2306 (2010).

${ }^{21}$ J. Berner, T. Jung, and T. Palmer, "Systematic model error: The impact of increased horizontal resolution versus improved stochastic and deterministic parameterizations," J. Clim. 25, 4946-4962 (2012).

${ }^{22}$ S. Ha, J. Berner, and C. Snyder, "A comparison of model error representations in mesoscale ensemble data assimilation," Mon. Weather Rev. 143, 3893-3911 (2015)

${ }^{23}$ I. Grooms, Y. Lee, and A. Majda, "Ensemble filtering and low-resolution model error: Covariance inflation, stochastic parameterization, and model numerics," Mon. Weather Rev. 143, 3912-3924 (2015).

${ }^{24}$ P. Houtekamer, H. L. Mitchell, and X. Deng, "Model error representation in an operational ensemble Kalman filter," Mon. Weather Rev. 137, 2126-2143 (2009).

${ }^{25}$ D. Zupanski and M. Zupanski, "Model error estimation employing an ensemble data assimilation approach," Mon. Weather Rev. 134, 1337-1354 (2006).

${ }^{26}$ I. Grooms and A. J. Majda, "Stochastic superparameterization in quasigeostrophic turbulence," J. Comput. Phys. 271, 78-98 (2014).

${ }^{27}$ I. Grooms and L. Zanna, "A note on 'toward a stochastic parameterization of ocean mesoscale eddies,"” Ocean Model. 113, 30-33 (2017).

${ }^{28}$ A. F. Thompson and W. R. Young, "Scaling baroclinic eddy fluxes: Vortices and energy balance," J. Phys. Oceanogr. 36, 720-738 (2006).

${ }^{29}$ S. Williams, M. Petersen, P.-T. Bremer, M. Hecht, V. Pascucci, J. Ahrens, M. Hlawitschka, and B. Hamann, "Adaptive extraction and quantification of geophysical vortices," IEEE Trans. Visualization Comput. Graphics 17, 2088-2095 (2011).

${ }^{30}$ S. Keating, A. Majda, and K. Smith, "New methods for estimating ocean eddy heat transport using satellite altimetry," Mon. Weather Rev. 140, 1703-1722 (2012).

${ }^{31}$ M.-I. Pujol, Y. Faugère, G. Taburet, S. Dupuy, C. Pelloquin, M. Ablain, and N. Picot, "Duacs dt2014: The new multi-mission altimeter dataset reprocessed over 20 years," Ocean Sci. 12, 1067-1090 (2016).

${ }^{32} \mathrm{Y}$. Xu and L.-L. Fu, "The effects of altimeter instrument noise on the estimation of the wavenumber spectrum of sea surface height," J. Phys. Oceanogr. 42, 2229-2233 (2012).

${ }^{33}$ C. Dufau, M. Orsztynowicz, G. Dibarboure, R. Morrow, and P.-Y. Le Traon, "Mesoscale resolution capability of altimetry: Present and future," J. Geophys. Res. 121, 4910-4927, https://doi.org/10.1002/2015JC010904 (2016).

${ }^{34}$ J. Gould, D. Roemmich, S. Wijfells, H. Freeland, I. Ignaszewsky, X. Jianping, S. Pouliquen, Y. Desaubies, U. Send, K. Radhakrishnan, K. Takeuchi, K. Kim, M. Danchenkov, P. Sutton, B. King, B. Owens, and S. Riser, "Argo profiling floats bring new era of in situ ocean observations," EOS 85, 185 (2004).

${ }^{35}$ The term "square exponential" is used here to distinguish a correlation function from a Gaussian probability density.

${ }^{36}$ Results degrade for a smaller correlation length $L_{c}=60 \mathrm{~km}$, and are relatively insensitive for a larger correlation length $L_{c}=150 \mathrm{~km}$.

${ }^{37}$ T. Gneiting, W. Kleiber, and M. Schlather, "Matérn cross-covariance functions for multivariate random fields," J. Am. Stat. Assoc. 105, 1167-1177 (2010). 